\title{
VARIACIONES EN LA RIQUEZA DE MACROMAMÍFEROS DE ESPAÑA DURANTE EL NEÓGENO
}

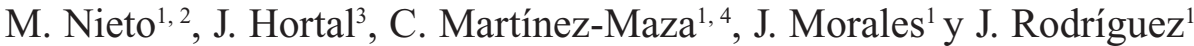

\begin{abstract}
RESUMEN
La composición y abundancia de las faunas de macromamíferos de la Península Ibérica ha sufrido importantes cambios durante el Neógeno (23-2 millones de años antes del presente). En el presente artículo se analizan y describen estos cambios, discutiéndose las faunas implicadas y apuntándose posibles causas de los cambios observados. Los resultados muestran un fuerte aumento en el número de especies presentes en la Península desde el final del Mioceno inferior hasta la primera mitad del Mioceno tardio, seguido de un fuerte descenso hasta el Plioceno, interrumpido por un aumento temporal de la riqueza durante el final del Mioceno. Paralelamente a la riqueza general de la Península, se aprecia que las faunas locales (correspondientes a yacimientos individuales) registradas durante el Mioceno medio y superior son, en algunos casos, excepcionalmente diversas, equiparables a las de los ecosistemas tropicales más ricos de la actualidad. Los cambios temporales en la diversidad son comúnmente interpretados como respuestas a cambios climáticos. Sin embargo, los análisis realizados apuntan la posibilidad de que muchos de estos cambios sean consecuencia de diferencias en el registro. A nivel de diversidad local, por diferencias en los procesos de formación de los yacimientos y, a nivel regional (de la Península Ibérica), relacionados con el número de yacimientos y áreas estudiadas en los distintos momentos. Asimismo, la comparación de los patrones de diversidad obtenidos con curvas climáticas globales publicadas recientemente, así como con distintos eventos biogeográficos relacionados con la apertura y cierre de vías migratorias, permiten apreciar una mayor relación de los cambios de diversidad con procesos biogeográficos y en cierto grado con las variaciones en los niveles globales de $\mathrm{CO}_{2}$. Los resultados apuntan también diversos patrones de relación entre la diversidad local (el número de especies por yacimiento) y la regional (el número de especies en la Península Ibérica) que podrían estar relacionados con cambios en la heterogeneidad ambiental presente en la Península a lo largo del tiempo.
\end{abstract}

Palabras clave: Riqueza de especies, Neógeno, Macromamíferos, España, Registro fósil, Cambio ambiental, Heterogeneidad.

\section{ABSTRACT \\ Changes in the macromammal richness of Spain during the Neogene}

Abundance and composition of Iberian macromammal faunas has suffered important changes during the Neogene ( 23 to 2 million years before present). The present paper analizes and describes these changes, also disccusing the taxa involved and their possible causes. Results from the present study point out the existence of an increase in the Iberian species richness from the end of the Lower Miocene to the first half of the Upper Miocene, followed by an strong impoverishment from then on till the Pliocene, interrupted by a temporal increase during the end of the Miocene. Local faunas (individual localities) from the Middle and Upper Miocene are, in some cases, exceptionally diverse, comparable to the richest extant tropical ecosystems. Diversity changes in time are usually explained as a response to climatic changes. However, our analyses point out that many of the observad changes in diversity may be due to differences in the fossil record quality. At locality (local) level caused by differences in the processes of locality formation, while at regional scale due to the number of deposits

Departamento de Paleobiología. Museo Nacional de Ciencias Naturales. CSIC. Madrid. E-mail: mcnd153@mncn.csic.es Área de paleontología. Dpto. Ciencias de la Tierra. Universidad de Zaragoza. Zaragoza. España.

Departamento de Biodiversidad y Biología Evolutiva. Museo Nacional de Ciencias Naturales. CSIC. Madrid.

Graduate School of Art and Science, Harvard University, Massachussets, U.S.A. 


\begin{abstract}
and areas studied in each time lapse. Comparison of the obtained patterns of diversity change with recently published global climate curves for the Neogene as well as with the up to date biogeographic knowledge, shows that diversity changes appear to be most closely related with the open and close of biogeographic barriers and to a lesser degree of variation in the global levels of $\mathrm{CO}_{2}$ concentration. Our results also show different relationship between local (number of species per site) and regional (number of species in the whole Iberian Peninsula) diversity that could be due to changes in the environmental heterogeneity of the Iberian Peninsula along the time considered.
\end{abstract}

Keywords: Species richness, Macromammals, Neogene, Spain, Fossil record, Environmental changes, Heterogeneity.

\section{Introducción}

Conocidos desde mediados del siglo XVIII, los yacimientos neógenos con mamíferos son excepcionalmente abundantes en España. Hasta la fecha, se han descubierto y estudiado más de 200 yacimientos con macromamíferos repartidos en varias cuencas continentales, abarcando la totalidad del rango temporal del Neógeno. La riqueza y relevancia de estas series ha propiciado la definición de alguno de los principales pisos continentales del Neógeno europeo, aceptados internacionalmente, caso del Vallesiense (Mioceno), cuyo estratotipo se encuentra en la cuenca del VallésPenedés (Cataluña), o de los pisos miocenos Turoliense, Aragoniense y Rambliense y el plioceno Alfam-briense, con estratotipos en la cuenca de Calatayud-Teruel (Aragón).

Los estudios taxonómicos, con descripción de nuevos taxones, son abundantes y durante las últimas décadas del pasado siglo se han llevado a cabo importantes revisiones de los principales grupos de macromamíferos. La información resultante ha permitido elaborar una importante base de datos referente a la diversidad presente en nuestro pais durante el Neógeno, parcialmente publicada en Azanza et al. (1997), Fraile et al. (1997), Nieto et al. (1997), Sánchez et al. (1998) y Morales et al. (2000).

Estudios previos describiendo los cambios en la diversidad existente en nuestro pais a lo largo del Neógeno (por ejemplo Morales \& Nieto, 1997; Morales et al., 1999) coinciden en la existencia de variaciones importantes en la riqueza global de especies, con un fuerte aumento durante el Mioceno medio y tardio y un fuerte descenso al final de este periodo y durante el Plioceno (ver, por ejemplo, Morales \& Nieto, 1997). Diversos estudios realizados durante los últimos años han demostrando la importancia de la información proporcionada por las faunas de mamíferos tanto a la hora de inferir diversos parámetros ambientales del pasado (Meulen \& Daams, 1992) como para evaluar los efectos de cambios climáticos o biogeográ- ficos en la estructura y riqueza de las comunidades (Pickford y Morales, 1994; Janis et al., 2002)

En el presente trabajo se presentan datos actualizados de la riqueza de macromamíferos de España en el Neógeno y se analizan de forma preliminar las tendencias y cambios registrados a lo largo de este periodo. Asimismo, se evalua el efecto que puedan tener las diferencias en el conocimiento de las distintas etapas del Neógeno sobre las estimaciones de la riqueza y sus cambios a lo largo del tiempo.

\section{Métodos}

\section{DATOS FAUNísTICOS}

Los datos empleados en el presente estudio son una actualización de los publicados por Cerdeño (1992), Alcalá (1994), Azanza et al. (1997), Fraile et al. (1997), Made (1997), Nieto et al. (1997) con las recientes revisiones publicadas por Sánchez et al. (1998), Aguirre \& Rabano (1999), Morales et al. (2000), Dam et al. (2001), incluyendo datos de yacimientos todavía sin publicar. La base de datos obtenida incluye 190 yacimientos con 343 especies de macromamíferos. Bajo el término macromamíferos se han incluido las especies pertenencientes a los ordenes Artiodactyla, Perissodactyla, Hyracoidea, Proboscidea, Primates, Creodonta y Carnivora. La taxonomía sigue la publicada por Mazo (1977) para los proboscideos, Alcalá (1994) para Hyracoidea, Cerdeño \& Nieto (1995) para Rhinocerotidae, Azanza et al. (1997) para Cervoidea, Fraile et al. (1997) para Carnivora y Creodonta, Made (1997) para Suidae, Nieto et al. (1997) para Bovoidea, Alberdi (1989) para los équidos hiparioninos y Sánchez et al. (1998) para los équidos anquiterinos.

Cada yacimiento ha sido localizado temporalmente siguiendo la biocronología basada en unidades mamífero MN (Mammal Neogene; intervalos de tiempo caracterizados por un determinado conjunto de especies) originalmente propuesta por 
Mein (1975) y en las posteriores actualizaciones realizadas (ver Agustí, 1999). Los limites temporales de las distintas unidades siguen los propuestos por Steininger y colaboradores (Steininger et al., 1996). Cada uno de los yacimientos incluidos en la base de datos ha sido localizado dentro de una de las siguientes cuencas terciarias: (1) Ebro; (2) Tajo; (3) Duero; (4) Calatayud-Teruel; (5) VallésPenedés; (6) Levantinas; (7) Béticas.

\section{CAlculo de la RiQueza de especies}

La riqueza se ha estimado para el total de especies de macromamíferos y para el total de especies de ungulados. Este último dato se ha incluido en los análisis, por una parte, por oposición a los carnívoros, frecuentemente escasos y mal representados en los yacimientos y, por otra parte, dada la fuerte relación de los ungulados con la vegetación y, por tanto, con el hábitat (Vrba, 1980; Janis et al., 2002).

El empleo de especies como unidades taxonómicas puede ser problemático para comparar la diversidad observada en distintas áreas o lapsos temporales. Este riesgo está causado por el carácter arbitrario de las especies, dependiente de los criterios de los autores de las mismas, de forma que el número de especies en un área o edad depende del criterio más o menos conservador de los autores a la hora de definir nuevas especies. En este sentido se ha propuesto que el uso de géneros como una posible solución, más conservadora. Sin embargo, el presente estudio está basado en revisiones globales de las faunas, de forma que los distintos grupos taxonómicos han sido revisados por un mismo autor o grupo de trabajo en toda la Península a lo largo de todo el lapso temporal considerado. Ello supone que el criterio a la hora de definir especies es coherente a lo largo del tiempo y el espacio considerado dentro de los distintos grupos. No ocurre lo mismo a la hora de comparar entre taxa, es decir, los criterios a la hora de definir especies en los diferentes grupos no son comparables pero, en este sentido, el empleo de géneros no soluciona el problema dado que su definición tambien es arbitraria, incluso más marcadamente a este nivel. En este sentido, el problema derivado del empleo de especies como unidades se circunscribe al reconocimiento de la variación intraespecífica frente a la interespecífica mientras que el caso de los géneros su definición es completamente arbitraria, dependiendo completamente de grados evolutivos de dificil definición e imposibles de comparar entre los diferentes grupos.

Los estimadores de la diversidad que hemos empleado han sido:
1. El número de especies registradas en el conjunto de la Península Ibérica en cada biozona. Estas estimaciones pueden considerarse equivalentes a los inventarios regionales realizados con especies actuales, aunque difieren por la combinación de datos provenientes de un lapso más o menos largo de tiempo, en cualquier caso muy superiores a los planteables para la actualidad. Dado que la duración temporal de los intervalos considerados (MN zones) difiere fuertemente entre las distintas biozonas, hemos empleado análisis de regresión para analizar el efecto de la duración de la biozona sobre la riqueza observada. Un aspecto fundamental a considerar es el relativo al muestreo de cada biozona. Resulta evidente que la acumulación de yacimientos supone un incremento creciente del conocimiento de la diversidad existente en cada biozona. La evaluación del efecto del número de yacimientos considerados sobre la riqueza estimada se ha analizado mediante curvas de rarefacción, tal y como se describe más adelante. Otro factor, relacionado con el anterior, que puede ser relevante es el concerniente a la heterogeneidad presente en la Península. Las diferencias ambientales o biogeográficas entre las cuencas muestreadas pueden conllevar la existencia de faunas diferentes en ellas. Este aspecto ha sido ya planteado para la Península Ibérica por Morales et al. (1999) entre otros y tiene como consecuencia diferencias en la riqueza de especies observada en la Península dependiendo de las cuencas en las que existe registro. Este efecto se ha analizado de forma preliminar por medio de regresiones del número de especies registradas en la península frente al número de cuencas presentes en cada biozona.

2. El número de especies en yacimientos individuales. De igual manera que los estimadores precedentes pueden considerarse equivalentes a los inventarios regionales actuales, los cálculos de la riqueza en yacimientos individuales pueden considerarse equivalentes a estimadores de riquezas locales y como tales han sido previamente empleadas para estimar diversas características de las comunidades del pasado (Andrews et al., 1979; Janis et al., 2000; Rodríguez y Nieto, en preparación). Distintos factores relacionados con los procesos de formación de los yacimientos pueden contribuir a la imprecisión en la estimación de la riqueza de las comunidades originales. Por una parte, se puede subestimar el número total de especies si el yacimiento contiene una muestra escasa. Este problema se ha minimizado eligiendo yacimientos con muestras abundantes, superiores a varios cientos de ejemplares. Por otra 
Tabla 1.- Número de especies de macromamíferos en las distintas biozonas (MN) del Neógeno de España. MN= Mammal Neogene, biozona del Neógeno según la biocronología de Mein (1975). Ma= Edad media de la biozona, en millones de años antes de la actualidad. Dur.= Duración de la biozona en millones de años. Yac.= Número de yacimientos incluidos dentro de la biozona. Número de especies total (T) y de ungulados (H) en España (Total) y las diferentes cuencas: (1) Ebro; (2) Tajo; (3) Duero; (4) Calatayud-Teruel; (5) Vallés-Penedés; (6) Levantinas; (7) Béticas.

Table 1. - Number of macromammal species in the Neogene of Spain along the diferent mammalian stages (MN). MN= Mammal Neogene stage according to Mein (1975). Ma= Age of the mammalian stage, x10 years. Dur.= length of the biozone in megaanuum. Yac. $=$ Number of localities included in the stage. Total number of species $(\mathrm{T})$ and ungulates $(\mathrm{H})$ in all Spain (Total) and the different Tertiary basins: (1) Ebro; (2) Tajo; (3) Duero; (4) Calatayud-Teruel; (5) Vallés-Penedés; (6) Levantinas; (7) Béticas.

\begin{tabular}{|c|c|c|c|c|c|c|c|c|c|c|c|c|}
\hline $\mathrm{MN}$ & Ma & Dur. & Yac. & & otal & 1 & 2 & 3 & 4 & 5 & 6 & 7 \\
\hline \multirow[t]{2}{*}{17} & 2.275 & 0.65 & 10 & $\mathrm{~T}$ & 36 & & 1 & & 20 & & 11 & 11 \\
\hline & & & & $\mathrm{H}$ & 23 & & 1 & & 9 & & 8 & 11 \\
\hline \multirow[t]{2}{*}{16} & 3 & 0.8 & 4 & $\mathrm{~T}$ & 30 & 22 & 7 & & & & 10 & \\
\hline & & & & $\mathrm{H}$ & 18 & 10 & 7 & & & & 5 & \\
\hline \multirow[t]{2}{*}{15} & 3.8 & 0.8 & 6 & $\mathrm{~T}$ & 24 & & 21 & & 4 & & & \\
\hline & & & & $\mathrm{H}$ & 14 & & 11 & & 4 & & & \\
\hline \multirow[t]{2}{*}{14} & 4.75 & 1.1 & 7 & $\mathrm{~T}$ & 29 & & & & 28 & & & 1 \\
\hline & & & & $\mathrm{H}$ & 21 & & & & 20 & & & 1 \\
\hline \multirow[t]{2}{*}{13} & 6.2 & 1.8 & 12 & $\mathrm{~T}$ & 58 & & & & 33 & & 34 & 9 \\
\hline & & & & $\mathrm{H}$ & 34 & & & & 18 & & 22 & 9 \\
\hline \multirow[t]{2}{*}{12} & 7.65 & 1.1 & 12 & $\mathrm{~T}$ & 45 & & & & 44 & & 5 & \\
\hline & & & & $\mathrm{H}$ & 21 & & & & 20 & & 5 & \\
\hline \multirow[t]{2}{*}{11} & 8.6 & 0.8 & 7 & $\mathrm{~T}$ & 37 & & & & 24 & 15 & 20 & \\
\hline & & & & $\mathrm{H}$ & 23 & & & & 15 & 11 & 13 & \\
\hline \multirow[t]{2}{*}{10} & 9.25 & 0.5 & 15 & $\mathrm{~T}$ & 51 & & 16 & & 21 & 34 & & \\
\hline & & & & $\mathrm{H}$ & 34 & & 7 & & 15 & 26 & & \\
\hline \multirow[t]{2}{*}{9} & 10.15 & 1.7 & 20 & $\mathrm{~T}$ & 78 & & 3 & 27 & 13 & 61 & & \\
\hline & & & & $\mathrm{H}$ & 44 & & 3 & 14 & 10 & 35 & & \\
\hline \multirow[t]{2}{*}{$7 / 8$} & 11.85 & 1.3 & 19 & $\mathrm{~T}$ & 55 & & & 22 & 22 & 29 & & \\
\hline & & & & $\mathrm{H}$ & 35 & & & 13 & 15 & 19 & & \\
\hline \multirow[t]{2}{*}{6} & 13.85 & 2.7 & 15 & $\mathrm{~T}$ & 35 & 2 & 28 & & 22 & 2 & & \\
\hline & & & & $\mathrm{H}$ & 24 & 2 & 21 & & 15 & 2 & & \\
\hline \multirow[t]{2}{*}{5} & 16.1 & 1.8 & 26 & $\mathrm{~T}$ & 49 & 12 & 41 & & 12 & 1 & & \\
\hline & & & & $\mathrm{H}$ & 34 & 9 & 28 & & 11 & 1 & & \\
\hline \multirow[t]{2}{*}{4} & 17.5 & 1 & 21 & $\mathrm{~T}$ & 52 & 2 & 18 & & 19 & 20 & 21 & \\
\hline & & & & $\mathrm{H}$ & 37 & 2 & 17 & & 10 & 20 & 14 & \\
\hline \multirow[t]{2}{*}{3} & 19 & 2 & 9 & $\mathrm{~T}$ & 20 & & & & 13 & 12 & & \\
\hline & & & & $\mathrm{H}$ & 19 & & & & 12 & 12 & & \\
\hline \multirow[t]{2}{*}{2} & 21.4 & 2.8 & 7 & $\mathrm{~T}$ & 28 & & 20 & 13 & 3 & & & \\
\hline & & & & $\mathrm{H}$ & 21 & & 14 & 10 & 3 & & & \\
\hline
\end{tabular}

parte, diversos procesos tafonómicos (procesos de fosilización) pueden alterar la composición del yacimiento y dar lugar a asociaciones que no son representativas de las originales. Así, por ejemplo, es posible sobreestimar el número de especies coexistentes si el periodo de tiempo representado en el yacimiento contiene cambios en las comunidades de mamíferos y, por tanto, es una mezcla de distintas faunas ("time-averaging"). En este sentido, hemos intentado incluir yacimientos suficientemente estudiados desde el punto de vista tafonómico, sin sesgos marcados (por ejemplo en las tallas o grupos representa- dos) y cuyas asociaciones puedan considerarse representativas de las originales, sin mezclas temporales apreciables. Sin embargo, también se han incorporado yacimientos con un menor control tafonómico para completar el muestreo, en la confianza de que no existe razón alguna para esperar que los sesgos tafonómicos difieran de forma sistemática entre unas biozonas y otras (Janis et al., 2002).

De esta manera, asumiendo que las biozonas representan periodos donde la fauna mantiene una relativa estabilidad en su composición (ver sin embargo, Azanza et al., 1997), podemos utilizar los 
tres componentes en los que Whittaker (1960) divide la biodiversidad para describir los patrones que aparecen en cada biozona, teniendo en cuenta que nos referimos a comunidades durante lapsos prolongados de tiempo, en lugar de a comunidades ecológicas actuales, es decir, al efecto de una grabación frente al de una instantánea. Los componentes de la biodiversidad enunciados por Whittaker han sido utilizados para multitud de trabajos utilizando definiciones ad hoc, por lo que su definición puede ser confusa, siendo necesario identificar claramente en cada trabajo cuál es el significado exacto de los términos aplicados (ver Whittaker et al., 2001 y Koleff \& Gaston, 2002). En este trabajo definimos:

- $\alpha$-diversidad, o diversidad local, como el número de especies que encontramos en un yacimiento, asumiendo que son una muestra de la fauna perteneciente a los diferentes paisajes localizados alrededor del yacimiento durante el tiempo en el que este se está formando.

- $\gamma$-diversidad, o diversidad regional, como el número total de especies presentes en el inventario de toda la Península durante esa biozona, asumiendo que es una muestra del tamaño del pool total de especies 'disponibles' para instalarse en las comunidades locales a lo largo de la biozona correspondiente.

- $\boldsymbol{\beta}$-diversidad, o reemplazo, equivale al recambio de especies entre los diferentes yacimientos de cada biozona, relacionando el tamaño del pool de especies ibérico en cada momento con la riqueza de las comunidades locales representadas por los yacimientos.

\section{CURVAS DE RAREFACCIÓN}

Como se ha señalado anteriormente, la riqueza observada en la Península Ibérica en cada biozona depende del número de yacimientos registrados. En general, cualquier medida de riqueza, considerada como un indicador de la cantidad de especies en una asociación, depende del tamaño muestral. Cuanto mayor sea el número de muestras (número de yacimientos en nuestro caso) mayor será el número de especies registrado, hasta llegar a la asíntota o techo, marcada por el número total de especies presentes en el área en estudio. Con el objeto de determinar el grado de conocimiento que poseemos del pool total de especies de cada biozona ( $\gamma$-diversidad), utilizamos curvas de rarefacción (ejemplos en Wing et al., 1995; Alroy, 1996). En ellas se relaciona el hallazgo de especies nuevas para el inventario de una determinada $\mathrm{MN}$ con el número de yacimien-
Tabla 2.- Número de especies de macromamíferos en los yacimientos de las distintas biozonas (MN) del Neógeno de España. MN= Mammal Neogene, biozona del Neógeno según la biocronología de Mein (1975). Yacimiento= denominación del yacimiento (en orden alfabético dentro de cada biozona). $\mathrm{Ma}=\mathrm{Edad}$ media de la biozona, en millones de años antes de la actualidad. Cuenca= (1) Ebro; (2) Tajo; (3) Duero; (4) Calatayud-Teruel; (5) Vallés-Penedés; (6) Levantinas; (7) Béticas. Herb=Número de especies de ungulados. Total $=$ Número de especies total.

Table 2.- Number of macromammal species in selected spanish localities along the diferent mammalian stages (MN) of the Neogene. $\mathrm{MN}=$ Mammal Neogene stage according to Mein (1975). Ma $=$ Age of the mammalian stage, x10 $10^{6}$ years. Yacimiento $=$ Locality name (in alfabetic order). Cuenca $=$ basin where the locality is placed; (1) Ebro; (2) Tajo; (3) Duero; (4) Calatayud-Teruel; (5) Vallés-Penedés; (6) Levantinas; (7) Beticas. Herb $=$ number of ungulate species; Total $=$ total number of macromamal species.

\begin{tabular}{|c|c|c|c|c|c|}
\hline $\mathrm{MN}$ & $\mathrm{Ma}$ & Yacimiento & Cuenca & Herb & Total \\
\hline \multirow[t]{2}{*}{17} & 2.275 & Huélago & 4 & 10 & \\
\hline & & Puebla de Valverde & 7 & 9 & 20 \\
\hline \multirow[t]{2}{*}{16} & 3 & Rincón & 6 & 5 & 10 \\
\hline & & Villarroya & 1 & 10 & 22 \\
\hline \multirow[t]{2}{*}{15} & 3.8 & Las Higueruelas & 2 & 6 & \\
\hline & & Layna & 2 & 7 & 17 \\
\hline \multirow[t]{2}{*}{14} & 4.75 & La Calera & 4 & 8 & 15 \\
\hline & & La Gloria 4 & 4 & 8 & 12 \\
\hline \multirow[t]{4}{*}{13} & 6.2 & El Arquillo & 4 & 15 & 22 \\
\hline & & Las Casiones & 4 & 13 & 22 \\
\hline & & Milagros & 4 & 8 & 11 \\
\hline & & Venta del Moro & 6 & 13 & 24 \\
\hline \multirow[t]{2}{*}{12} & 7.65 & Cerro de la Garita & 4 & 18 & 31 \\
\hline & & Los Mansuetos & 4 & 10 & 21 \\
\hline \multirow[t]{3}{*}{11} & 8.6 & Crevillente 2 & 6 & 13 & 20 \\
\hline & & Piera & 5 & 11 & 15 \\
\hline & & Puente Minero & 4 & 10 & 19 \\
\hline \multirow[t]{4}{*}{10} & 9.25 & La Roma 2 & 4 & 10 & 12 \\
\hline & & Masia del Barbo 2B & 4 & 7 & 9 \\
\hline & & Sant Miquel de Taudell & 5 & 6 & 11 \\
\hline & & Villadecabals & 5 & 18 & 21 \\
\hline \multirow[t]{4}{*}{9} & 10.15 & Can Llobateras & 5 & 20 & 40 \\
\hline & & Can Ponsic & 5 & 18 & \\
\hline & & Hostalets de Pierola Superior & 5 & 9 & 17 \\
\hline & & Valles de Fuentidueña & 3 & 14 & 27 \\
\hline \multirow[t]{2}{*}{$7 / 8$} & 11.85 & Hostalets de Pierola Inferior & 5 & 18 & 27 \\
\hline & & Toril 3 & 4 & 10 & 14 \\
\hline \multirow[t]{3}{*}{6} & 13.85 & Arroyo del Val & 4 & 14 & 19 \\
\hline & & Paracuellos 3 & 2 & 12 & 16 \\
\hline & & Paracuellos 5 & 2 & 9 & 12 \\
\hline \multirow[t]{4}{*}{5} & 16.1 & La Hidroeléctrica & 2 & 14 & \\
\hline & & Puente de Vallecas & 2 & 9 & 11 \\
\hline & & Retama & 2 & 9 & 16 \\
\hline & & Tarazona & 1 & 9 & 12 \\
\hline \multirow[t]{4}{*}{4} & 17.5 & Artesilla & 4 & 6 & 14 \\
\hline & & Buñol & 6 & 14 & 21 \\
\hline & & Córcoles & 2 & 13 & \\
\hline & & Els Cassots & 5 & 9 & \\
\hline \multirow[t]{3}{*}{3} & 19 & Agreda & 4 & 4 & 5 \\
\hline & & Molí Calopa & 5 & 6 & 6 \\
\hline & & Rubielos de Mora & 4 & 6 & 6 \\
\hline \multirow[t]{3}{*}{2} & 21.4 & Cetina de Aragon & 3 & 10 & 13 \\
\hline & & Loranca 1 & 2 & 12 & 18 \\
\hline & & Valquemado & 2 & 7 & \\
\hline
\end{tabular}




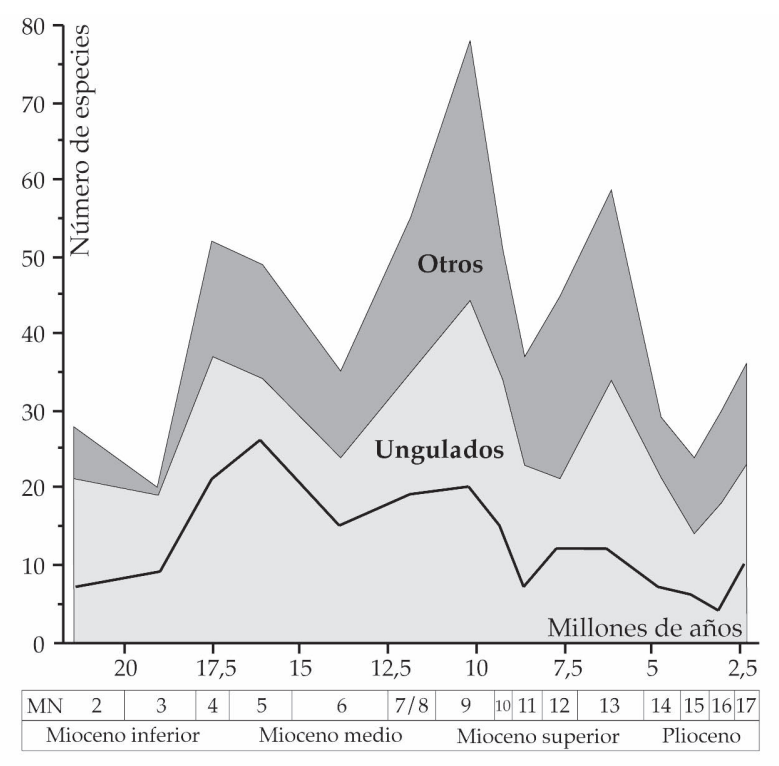

Fig. 1.- Riqueza de grandes mamíferos en las distintas biozonas del Neógeno de España. En trama clara: especies de ungulados; trama oscura: resto de macromamíferos; linea continua: número de yacimientos en la biozona.

Fig. 1.- Macromammal richness across the Neogene stages in Spain. Light gray $=$ number of ungulate species; dark gray $=$ rest of macromammal species; continuous line: number of localities in each biozone.

tos excavados, asumiendo que estos han actuado como muestreos de parte de la diversidad presente en ella, a la manera de las trampas o transectos de la actualidad (curvas de acumulación de especies; Soberón \& Llorente, 1993; Colwell \& Coddington, 1994; Gotelli \& Colwell, 2001). Por ello, para obtener estas curvas hemos definido como 'unidad muestral' el yacimiento, y como 'esfuerzo de muestreo', el número de yacimientos excavados en cada biozona (MN). La pendiente de la curva en cada punto es la tasa de encuentro de especies por yacimiento nuevo, una medida indirecta de la calidad del inventario. Cuanto menor sea la pendiente tras incorporar todos los yacimientos, más difícil es que un nuevo yacimiento añada especies no registradas en la biozona. Como la forma de esta curva varía en función del orden en que se introduzcan las 'muestras', aleatorizamos previamente 50 veces este orden de entrada para obtener una curva de rarefacción 'ideal', suavizada, mediante el programa EstimateS 6.0 (Colwell, 2000), en un procedi- miento similar al bootstrap (remuestreo) utilizado por Wing et al. (1995). Después, para determinar la pendiente en el último yacimiento incluido, ajustamos una función de Clench a la relación entre el número de especies inventariadas y el de yacimientos en cada biozona:

$$
S_{(y)}=(a \cdot y) /[1+(b \cdot y)]
$$

donde $S_{(v)}$ es el número de especies encontrado por yacimiento (y); $a$ y $b$ son los parámetros de la función, que se ajustaron a los datos de cada curva mediante un método de Simplex \& Quasi-Newton (StatSoft, 2001). La pendiente (primera derivada) de esta curva en cada punto es:

$$
r_{(y)}=a /\left(1+(b \times y)^{2}\right)
$$

donde $r_{(v)}$ es la pendiente de la curva para un esfuerzo de muestreo $y$. Cuando $y$ es el número total de yacimientos conocidos hasta la fecha en esa $\mathrm{MN}$, el valor de la pendiente es la tasa actual de incorporación de nuevas especies a su inventario.

\section{Resultados}

\section{Diversidad duRANTE El NeÓGeno}

El estudio por biozonas de la diversidad total de especies muestra un patrón caracterizado por un aumento de la riqueza de especies a finales del Mioceno inferior, aproximadamente hace 17,5 millones de años (tabla 1; figura 1). Los valores altos en el número de especies registradas se mantienen hasta el final del Mioceno tardio y comienzos del Plioceno, cuando se registra un fuerte descenso. Dentro de este patrón general se observan diversas variaciones puntuales, fundamentalmente un descenso marcado de la diversidad durante la biozona MN 3 (19 Ma), seguido de un periodo de alta diversidad durante la MN 4 (17,5 Ma). Tras este pico no se registra otro descenso hasta la MN $6(13,85 \mathrm{Ma})$, seguido de un fuerte ascenso con cenit en la MN 9 (10,15 Ma), cuando se registran los máximos valores de todo el Neógeno. Posteriormente se registra un descenso de la diversidad, interrumpido por un fuerte incremento durante la MN 13 (5,7 Ma). A finales del Plioceno (MN 17, 2,3 Ma.) se registra un leve aumento de la diversidad aunque sin alcanzar cotas similares a las observadas durante el Mioceno.

La $\gamma$-diversidad (es decir, en el conjunto de la Península) de ungulados en las distintas biozonas sigue un patrón muy similar, marcado por un menor 


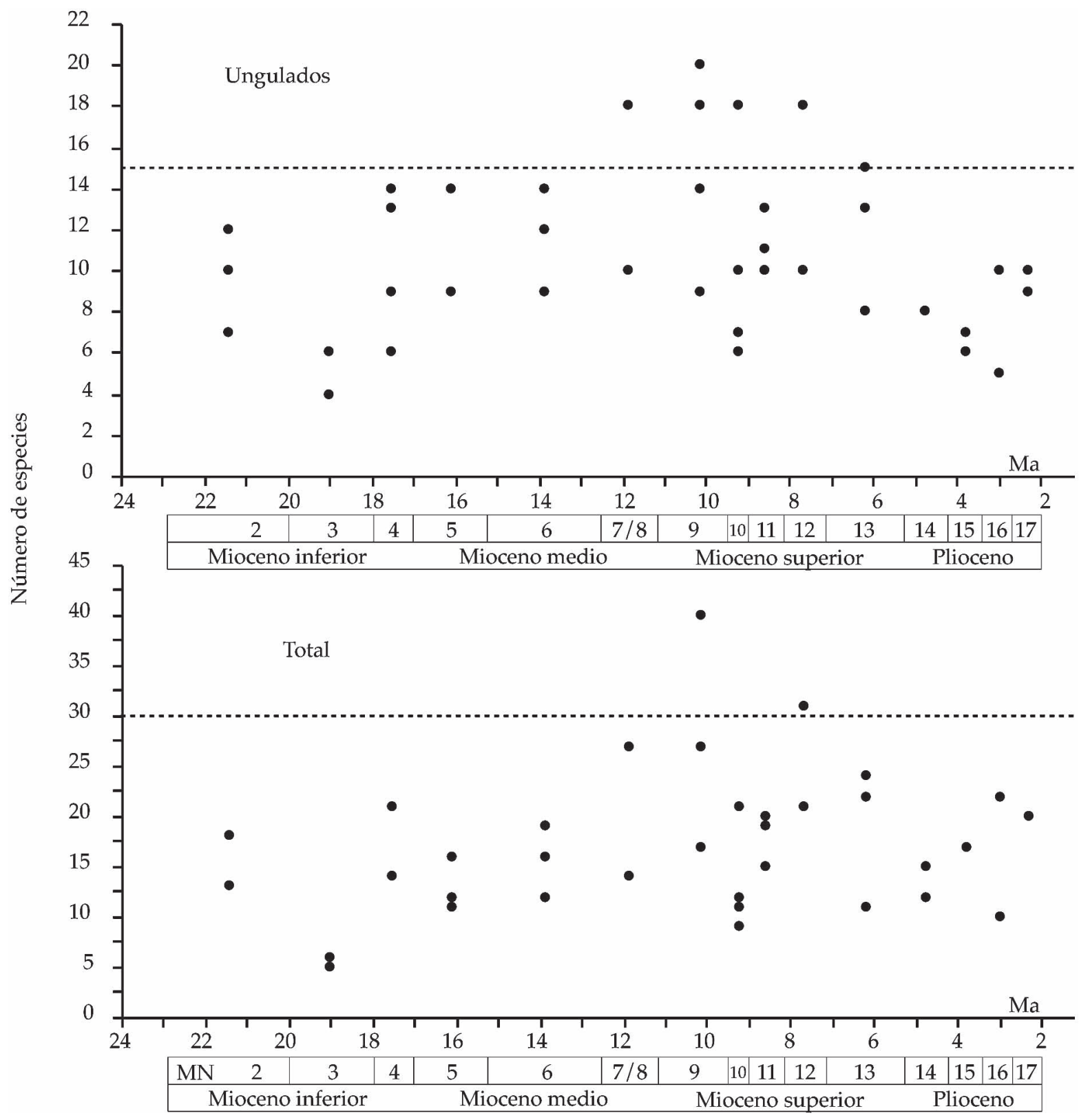

Fig. 2.- Variaciones en la abundancia de especies de ungulados (arriba) y total (abajo) en yacimientos seleccionados del Neógeno de España (datos en tabla 2). La línea discontinua corresponde al máximo de especies en comunidades actuales no tropicales.

Fig. 2.- Changes in ungulate (up) and total (down) species abundance in selected localities of the spanish Neogene (data in table 2). Discontinuous lines corresponds to the maximum number of species in recent non-tropical communities. 


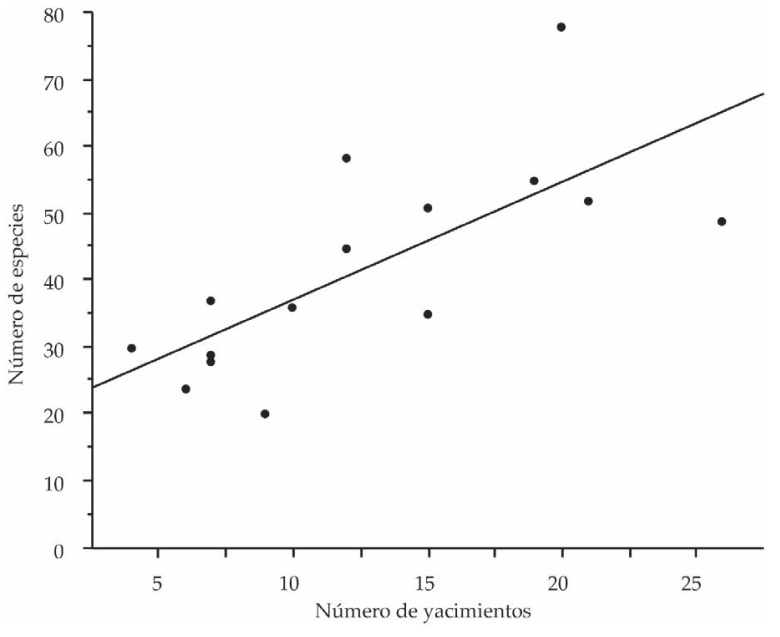

Fig. 3.- Relación entre la riqueza de especies y el número de yacimientos de las diferentes biozonas $(\mathrm{R}=0.72, \mathrm{p}<0.002)$.

Fig. 3.- Biplot of species richness and number of sites in the stages considered $(R=0.72, p<0.002)$.

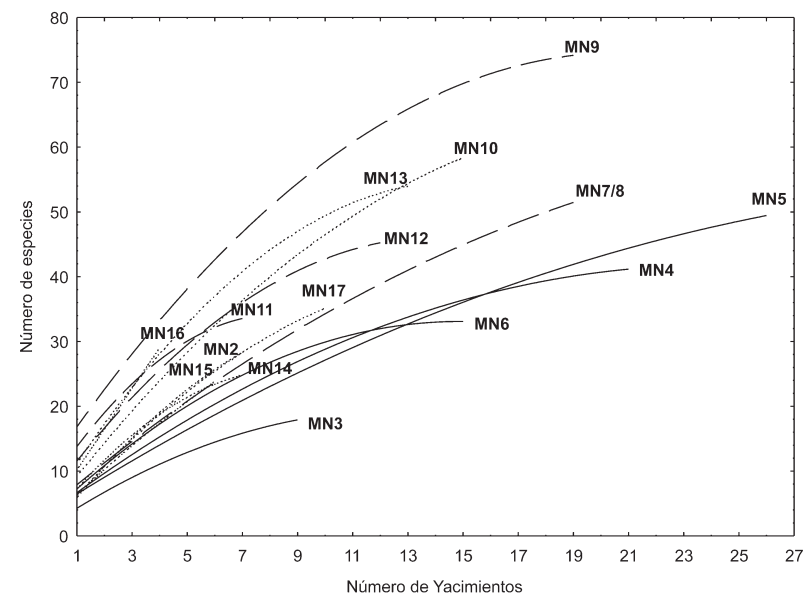

Fig. 4.- Curvas de rarefacción de las 15 biozonas (ver Tabla 3). El orden de entrada de los individuos en cada curva ha sido aleatorizado 50 veces. Línea continua: MNs con valores finales de pendiente entre 1 y 2 ; línea discontinua, entre 2 y 3 ; línea de puntos, más de 3 .

Fig. 4.- Rarefaction curves for the 15 stages (see table 3 ). The order of incussion of the indivuduals in the curve has been randomized 50 times. Continuous line: stages with slope values between 1 and 2; dashed line: slope values between 2 and 3; dotted line: slope values over 3 .
Tabla 3.- Curvas de rarefacción de las 15 biozonas (ver Figura 4). $S p p$ es el número de especies descubiertas, e $Y$ el de yacimientos excavados; $a$ y $b$ son los parámetros de la función de Clench ajustada a cada curva; $r_{(v)}$ es el valor de la pendiente en el último yacimiento; $S_{(y)}$ es la asíntota predicha por la ecuación de Clench. Todos los ajustes a la ecuación de Clench tuvieron porcentajes de varianza explicados superiores al 99\%.

Table 3.- Rarefaction curves of the considered stages (see figure 4). Spp corresponds to the number of recorded species; $Y$ to the number of localities; $a$ and $b$ are the parameters of the Clench function adjusted to each curve; $r_{(v)}$ is the slope value at the last site of the curve; $S_{(v)}$ is the predicted asimptote of the curve as predicted by the Clench function. All Clench functions adjustments have explained variance percentages over the $99 \%$.

\begin{tabular}{lcrrccr}
\hline Biozonas & $\boldsymbol{S} \boldsymbol{p p}$ & $\boldsymbol{Y}$ & $\boldsymbol{a}$ & $\boldsymbol{b}$ & $\boldsymbol{r}_{(\boldsymbol{v})}$ & $\boldsymbol{S}_{(\boldsymbol{v})}$ \\
\hline MN2 & 28 & 7 & 6.172 & 0.074 & 4.86 & 83.28 \\
MN3 & 18 & 9 & 4.221 & 0.125 & 1.86 & 33.79 \\
MN4 & 43 & 21 & 5.050 & 0.074 & 1.49 & 68.55 \\
MN5 & 50 & 26 & 3.918 & 0.041 & 1.83 & 95.26 \\
MN6 & 34 & 15 & 7.097 & 0.142 & 1.28 & 49.91 \\
MN7/8 & 54 & 19 & 5.330 & 0.052 & 2.68 & 101.69 \\
MN9 & 76 & 19 & 12.699 & 0.118 & 2.11 & 107.67 \\
MN10 & 59 & 15 & 7.558 & 0.063 & 4.02 & 120.78 \\
MN11 & 34 & 7 & 17.114 & 0.371 & 2.20 & 46.07 \\
MN12 & 46 & 12 & 10.821 & 0.156 & 2.40 & 69.30 \\
MN13 & 50 & 12 & 9.604 & 0.109 & 3.53 & 87.80 \\
MN14 & 25 & 7 & 8.156 & 0.184 & 3.07 & 44.32 \\
MN15 & 24 & 6 & 6.058 & 0.091 & 4.66 & 66.38 \\
MN16 & 29 & 4 & 10.362 & 0.112 & 8.64 & 92.70 \\
MN17 & 35 & 10 & 6.162 & 0.075 & 3.94 & 81.94 \\
\hline
\end{tabular}

incremento en el número de especies durante el Mioceno medio (figura 1). De manera similar, la diversidad registrada en la biozona MN 9 (10 Ma) no destaca respecto a la registrada en otros momentos del Neógeno, enmarcándose dentro de un nivel más homogéneo a lo largo de todo el Neógeno. Igualmente a lo observado en la $\gamma$-diversidad total, el final del Mioceno y comienzo del Plioceno marcan un descenso de la $\gamma$-diversidad de ungulados por debajo de los valores registrados durante el Mioceno. Esta tendencia se rompe al final del Plioceno (MN 16 y 17) con un aumento gradual del número de especies.

El cálculo de la diversidad en yacimientos individuales ( $\alpha$-diversidad) también coincide de forma general con lo observado en los análisis de riqueza peninsular en las biozonas (tabla 2; figura 2). Así, desde un inicial descenso durante la MN 3, se observa un aumento general de la $\alpha$-diversidad a partir de la MN 4 (17,5 Ma), que se mantiene hasta el final del Mioceno. De igual manera, se observa un máximo 


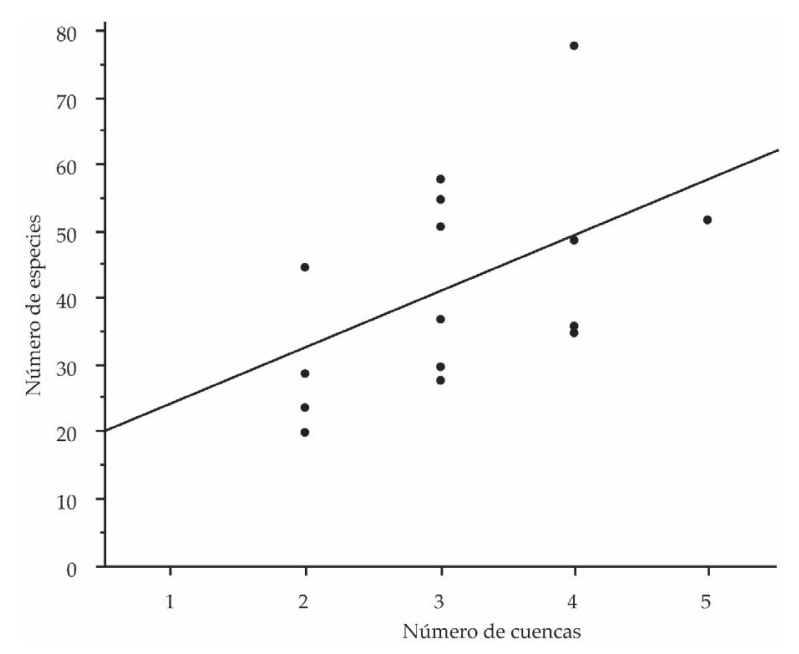

Fig. 5.- Relación entre la riqueza de especies y el número de cuencas con registro conocido en las diferentes biozonas $(\mathrm{R}=0.504, \mathrm{p}<0.056)$.

Fig. 5.- Biplot of species richness and number of basins with known record in the stages considered $(\mathrm{R}=0.504, \mathrm{p}<0.056)$.

de la riqueza de especies durante la $\mathrm{MN} 9(10 \mathrm{Ma})$ y un descenso importante durante el final del Mioceno hasta el Plioceno, con un descenso marcado durante las MN 10 y 11 (9-8 Ma). El patrón observado en el número de ungulados por yacimiento es asimismo similar, con una homogeneidad marcada a lo largo de todo el registro, solo interrumpida por los descensos registrados en el número de especies durante la MN 3 y al final del Mioceno (a partir de 5-6 Ma).

EFECTOS DEL MUESTREO SOBRE LOS VALORES OBSERVADOS DE RIQUEZA

La estrecha relación existente entre el número de yacimientos y la diversidad de cada biozona se aprecia en la figura 3. Las curvas de rarefacción de las 15 biozonas (Figura 4) indican que en todos los casos aún queda gran parte de la fauna fósil por descubrir y que las estimas de riqueza total de especies en cada biozona no son aún suficientemente precisas, siendo necesarias pendientes más bajas (inferiores a 0.01; ver discusión en Hortal \& Lobo, 2002). Así, ninguna biozona alcanzó pendientes inferiores a 1 (Tabla 3; figura 4), que indican una aproximación a un muestreo suficiente, en que todas las especies presentes han sido descubiertas. Sin embargo, cuatro MNs (3, 4, 5 y 6$)$ presentaron valores más bajos, entre 1 y 2 , y otras cuatro entre 2 y 3 (MNs 7/8, 9, 11, 12). En estas biozonas, con

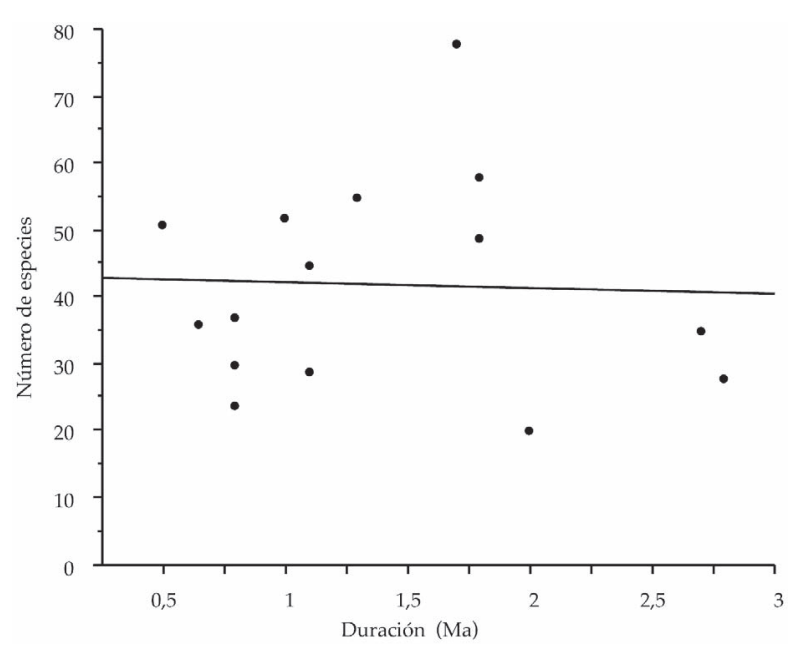

Fig. 6.- Relación entre riqueza específica y duración (en millones de años) de las diferentes biozonas $(R=-0.044 ; \mathrm{p}<0.877)$.

Fig. 6.- Biplot of species richness and length (in megannum) of the different stages $(\mathrm{R}=-0.044 ; \mathrm{p}<0.877)$.

un número relativamente pequeño de nuevos yacimientos se alcanzaría un nivel aceptable de fiabilidad en el inventario. Además, sí es posible discriminar estas últimas MNs en dos grandes grupos, definidos precisamente por sus pendientes. Las cuatro primeras, con pendientes menores de dos, son sensiblemente más pobres que las cuatro del segundo, por lo que con menor cantidad de yacimientos han alcanzado pendientes menores. Entre las biozonas con un muestreo más insuficiente se situan las MN 2, 10, 15, 16 y 17 cuyas curvas no presentan ningún atisbo de aproximación a la asíntota, tal y como demuestra la pendiente del último tramo de la curva de rarefacción. Entre ambos extremos se situan varias biozonas con valores de la pendiente altos pero más proximos a valores aceptables (MN 13 y 14).

Otro aspecto importante es la relación existente entre la abundancia de especies y el número de cuencas donde se dispone de registro en cada biozona. En la figura 5 se puede observar que existe una tendencia $(\mathrm{R}=0.504, \mathrm{p}<0.056)$ al aumento en el número de especies registradas en cada biozona con el aumento del número de cuencas con registro, aunque sin alcanzar niveles estadísticamente significativos $(p>0.05)$. La relación entre riqueza y número de cuencas es, sin embargo, compleja dado que las diferencias entre las cuencas varían de unas 
biozonas a otras, con momentos de mayor homogeneidad en el conjunto de la Península y con otros momentos más heterogéneos.

Un último factor que puede afectar a los valores de riqueza obtenidos en cada biozona es la duración temporal de la misma. Por analogía con los fenómenos de "time-averaging" en yacimientos individuales, podría esperarse un aumento de la diversidad con el incremento del lapso temporal cubierto por las biozonas. Sin embargo, como se aprecia en la figura 6 , se observa que no existe tal relación $(\mathrm{R}=-0.004, \mathrm{p}<0.877)$. Existe un motivo evidente para esta falta de correlación: las MN son biozonas definidas como lapsos temporales con una fauna de mamíferos común, de tal forma que la existencia de cambios en las faunas incluidas en una misma biozona de acuerdo con un gradiente temporal llevaría a la subdivisión de la biozona en nuevas biozonas.

\section{Discusión}

Los resultados obtenidos muestran importantes cambios en el número de especies de macromamíferos registrados en la Península Ibérica durante las distintas biozonas del Neógeno (tabla 1). Los momentos de mayor riqueza como la biozona MN 9 (aprox. $10 \mathrm{Ma}$ ) presentan valores 5 veces mayores que la riqueza registrada en los periodos más pobres como la MN 3 (aprox. 19 Ma). Sin embargo, estos resultados han de ser considerados con cautela. La relación observada entre el número de especies registradas y el número de yacimientos en las biozonas $(\mathrm{R}=0.745$, $\mathrm{p}<0.002$; figura 3) y los valores de las curvas de rarefacción (tabla 3) sugieren que las diferencias de riqueza observadas pueden ser consecuencia, al menos parcialmente, de diferencias en la calidad del registro, en forma del número de yacimientos conocidos.

Los análisis de rarefacción indican claramente que sólo una parte minoritaria de las biozonas puede considerarse suficientemente muestreada. Pero incluso las biozonas donde las curvas presentan valores terminales de la pendiente relativamente bajos, más proximos a la asíntota, la riqueza observada ha de ser considerada con cierta cautela, por cuanto en algunos casos el número de yacimientos y la representación espacial de la Península cubierta por los mismos es baja. La asíntota de una curva de acumulación de especies tan sólo indica el total de especies que estamos muestreando. Si nuestro muestreo en una biozona deter- minada (los datos de yacimientos con que contamos) está sesgado, ecológica o espacialmente, no recogemos toda la variabilidad de la diversidad de mamíferos presente en el área total de la Península y las curvas alcanzarán antes la asíntota pero sólo harán referencia al área o ambiente que hemos muestreado. Tal es el caso de la MN 3, la MN 6 y la $\mathrm{MN} 11$, todas ellas con un número yacimientos reducido ( 7 en el caso de la $\mathrm{MN} 11$ y 9 en la $\mathrm{MN}$ 3 ), repartidos en pocas cuencas y, por tanto, con falta de representación de faunas potencialmente distintas. La importancia de la heterogeneidad ambiental de España, causada por diferencias ambientales entre las cuencas, ha sido comentada previamente en Morales et al. (1999). Este aspecto se ve refrendado en nuestro estudio por la tendencia de la riqueza de la Península a aumentar con el incremento en el número de cuencas representadas en cada biozona $(\mathrm{R}=0.504, \mathrm{p}<0.056)$. La consecuencia para las curvas de rarefacción es que biozonas con pocas cuencas representadas pueden alcanzar valores de la pendiente bajas que sólo reflejan la saturación de la curva en la estimación de la riqueza de especies en las cuencas muestreadas, no así al total de la riqueza presente en el área total de la Península.

\section{VARIACIONES TEMPORALES EN LAS FAUNAS}

El Neógeno se inicia aproximadamente hace 23 millones de años, con un brusco cambio climático de breve duración denominado el "Miocene event" (Zachos et al., 2001). Europa Occidental se encuentra en este momento conectada a Asia únicamente por su parte más meridional y está completamente aislada de Africa. En España se registra un periodo de baja riqueza de especies durante las MN 2 y 3 (entre aproximandamente 23 y $18 \mathrm{Ma}$ ). Las curvas de rarefaccion indican que la primera de estas biozonas carece de un registro suficientemente completo mientras que la MN 3 sí está más o menos bien muestreada. Como se ha comentado previamente, los valores de la curva de rarefacción obtenidos en la MN 3 pueden considerarse, hasta cierto punto, artificiales, lo cual se ve refrendado por el hecho de que apenas si se cuenta con registro de carnívoros (1 especie, ver Fraile et al., 1997). Sin embargo, todo ello no debe oscurecer el hecho de que se trata de un periodo de relativa baja riqueza. Tanto la riqueza de especies total como la de ungulados es, en estos dos periodos, muy inferior a la registrada durante el Mioceno medio y superior (17,5 a $5 \mathrm{Ma})$. Tambien es inferior la riqueza de las comunidades individuales aunque en este caso las diferencias son menores. Estos valores sólo pueden 
considerarse preliminares en la MN 3 dada la carencia de yacimientos suficientemente muestreados. La fauna de estos primeros periodos del Mioceno es una continuación de las faunas existentes en el Oligoceno, aunque la falta de registro de este último periodo en la península impide asegurar esta afirmación. La fauna conocida está dominada por los rumiantes prepecora (sin apéndices craneales) que se encuentran en plena radiación, junto con un reducido número de especies de rinocerontes y suidos. Los carnívoros se encuentran dominados por formas de cánidos y úrsidos muy primitivos (Fraile et al., 1997) a los que se une un félido primitivo, Pseudaelurus, al final de la MN 2 (Alcalá et al., 1990). Estas faunas están, en algunos casos, en declive y el aislamiento parcial de Europa occidental (Rögl, 1999) no permite un intercambio fluido con regiones vecinas. Existe, no obstante, cierta comunicación con Asia desde donde llegan los primeros girafoideos, representados por el género Teruelia (Moyá-Solá \& Agustí, 1990).

Ya en la biozona MN 3 llega un inmigrante de origen norteamericano que ha atravesado toda Asia para llegar a Europa, el équido Anchitherium (Thenius, 1972), que caracterizará las faunas europeas hasta el Mioceno superior (11 Ma). Junto con este caballo tridáctilo aparecen en España los primeros ciervos y paleomerícidos (un grupo de rumiantes con apéndices craneales en los frontales y el occipital) fruto de la radiación Prepecora registrada en la biozona anterior (Azanza et al., 1997; Agustí, 1999). La MN 3 permite reconocer un fenómeno que será general en España a lo largo de gran parte del Neógeno: la diferenciación ecológica entre las cuencas orientales costeras (VallésPenedés y Levantinas) frente a las interiores (Tajo y Calatayud-Teruel, principalmente) (Agustí, 1999). Las primeras tendrán una influencia europea más marcada y un ambiente más húmedo $\mathrm{y}$ forestal mientras que las interiores serán más áridas por norma general.

La biozona MN4, hace aproximadamente 17,5 $\mathrm{Ma}$, marca el primer cambio fuerte en la riqueza de especies. Durante el millón de años cubierto por esta biozona, el número total de especies registradas en la Península prácticamente se duplica aunque la riqueza en los yacimientos individuales no supera en mucho la existente en los yacimientos del comienzo del Mioceno (MN 2). En conjunto, la MN 4 supone, en lo referente a los macromamíferos, el cambio de las faunas arcaicas del Oligoceno por las faunas propias del Mioceno. Se registran por primera vez varios taxa de origen africano, resultado del establecimiento temporal del denomi- nado "Gomphotherium landbridge" que comunicó Eurasia con África durante un tiempo aún por determinar (Rögl, 1999). Este cambio geográfico, acaecido durante la MN3 pero cuyos efectos sobre la fauna se aprecian fundamentalmente en la Península desde la MN 4, supone la entrada de los primeros proboscídeos (por ello conocido como el "Proboscidean event") y bóvidos de origen africano y la reaparición de algunos grupos de carnívoros como los creodontos (un grupo de carnívoros primitivos, Fraile et al., 1997). Este periodo también supone la diversificación de algunos grupos ya presentes en Europa occidental desde largo tiempo atras, como los rinocerontes que alcanzan una diversidad no igualada antes ni después en la Península (Cerdeño \& Nieto, 1995). En conjunto se aprecia un fuerte aumento de la riqueza peninsular relacionable con la apertura de vías migratorias de Europa con África, Asia y Norteamérica.

La biozona MN 5 ha recibido clasicamente en España el nombre de faunas con Hispanotherium (ver Morales et al., 1993), en razón del predominio de este rinoceronte de pequeño tamaño. Sus adaptaciones corredoras y su dieta pastadora, propias ambas de hábitats abiertos, de tipo sabana, han llevado a proponer un ambiente árido para esta época (Cerdeño, 1992; Cerdeño \& Nieto, 1995). Esta interpretación se ve confirmada por los datos polínicos (Sanz de Siria, 1985, 1988, 1994) y la fauna de micromamíferos (Agustí, 1999). Sin embargo, el estudio de diversas faunas de yacimientos especialmente significativos matiza esta visión (Morales et al., 1993), incorporando la presencia de hábitats boscosos a los hábitats abiertos para formar un mosaico de ambientes. Durante la MN 5 se registra un discreto descenso en la riqueza de especies presentes en la Península, atribuible fundamentalmente a la sustitución de la diversa fauna de rinocerontes de la biozona anterior por el mencionado Hispanotherium (Cerdeño y Nieto, 1995) mientras que el resto de la fauna mantiene su riqueza. En los yacimientos se observa un ligero descenso en la riqueza total de especies de difícil interpretación aunque el número de ungulados se mantiene prácticamente constante. Así, se puede considerar esta biozona como un periodo de estabilidad tras los fuertes cambios registrados en la biozona anterior, con cambios internos dentro de distintas lineas como la aparición de nuevas formas de bóvidos, suidos o Micromeryx, un pequeño artiodáctilo que va a estar presente en España hasta el Turoliense, hace menos de 9 millones de años (Agustí, 1999), así como la diversificación de los paleomerícidos (un grupo de rumiantes con apéndices craneales frontales y occipitales). 
Tras el fuerte aumento de la riqueza registrado anteriormente, la biozona MN 6 (aprox. $16 \mathrm{Ma}$ ) es una época de descenso en la riqueza de especies presentes en la Península. Sin embargo, este descenso puede estar relacionado con un registro más limitado, con ausencia de yacimientos significativos en zonas como la cuenca del Vallés-Penedés o las cuencas levantinas, ambas especialmente ricas en especies durante la MN 4. De hecho, en esta época se han descrito un alto número de especies endémicas de una u otra cuenca, por ejemplo en el caso del género Anchitherium (Sánchez et al., 1998; ver, sin embargo, Alberdi \& Rodríguez, 1999) y, por tanto, la riqueza de especies podría incrementarse de forma relevante con la incorporación de datos de otras cuencas. De forma coherente, la riqueza de especies en los yacimientos se mantiene en valores similares a los registrados en las biozonas previas. La MN 6 marca el declive de algunas formas muy características de las biozonas anteriores (caso de los paleomerícidos) y la entrada de los primeros hiénidos (procedentes de África; Fraile et al., 1997) o los artiodáctilos Tethytragus (Nieto et al., 1997) procedentes de Asia (Agustí, 1999). Asimismo, el característico rinoceronte Hispanotherium de la biozona anterior es reemplazado completamente por nuevas especies de rinocerontes (Cerdeño, 1992) y algo similar ocurre entre los cérvidos, con la entrada de los primeros Muntiacinae (Azanza et al., 1997). Se producen, pues, recambios de cierta importancia, con entrada de nuevos inmigrantes y una riqueza en los yacimientos que no parece concordar con un periodo de baja riqueza de especies para el conjunto de la Península, lo cual corrobora una vez más la posibilidad de una falta de registro como causa principal del descenso en la riqueza observada. Sin embargo, resulta sorprendente que las curvas de rarefacción obtenidas señalen esta biozona como la mejor muestreada (tabla 3 ) y con las estimas de la riqueza más fiables de todo el registro Neógeno. Aunque realmente el número de yacimientos conocidos es amplio, parece razonable que, tal y como hemos apuntado anteriormente, la falta de registro de cuencas significativas como el Vallés-Penedes sea la responsable de los bajos valores de riqueza y, al mismo tiempo, de los resultados obtenidos en las curvas de rarefacción, al no estar muestreando la totalidad de la Península.

La última biozona del Mioceno medio, correspondiente a la MN 7/8 (aprox. $12 \mathrm{Ma}$ ) supone la continuación de las faunas registradas anteriormente pero con un aumento en la riqueza a nivel peninsular hasta valores similares a las biozonas MN 4 y
5. Esta diferencia de riqueza con respecto a la biozona anterior podría deberse a la existencia, en esta biozona, de un buen registro de la cuenca del Vallés-Penedés. A pesar de ello, también es cierto que en la MN 7/8 también se registra un aumento en el número de especies por yacimiento (tanto totales como en número de ungulados) que apunta hacia un aumento de la diversidad a escala local y peninsular. Como ocurrirá a lo largo de todo el Neógeno, continúa la sustitución en las diferentes líneas de algunas especies por nuevos inmigrantes. En esta biozona se produce un fuerte cambio en los suidos y asimismo dentro de los bóvidos, con la entrada de los primeros representantes de los Boselaphini que dominarán las faunas de ungulados hasta los 9 millones de años (final de la $\mathrm{MN}$ 10). Una entrada relevante es la del primate Dryopithecus en la cuenca del Vallés-Penedés asociado con hábitats más forestados (Morales et al., 1999; Agustí, 1999).

El Mioceno superior se inicia en la biozona MN 9, hace 11,2 millones de años, con la entrada del équido tridáctilo Hipparion (Sen, 1990; Garces et al., 1997). Este inmigrante procedente de Norteamérica aprovecha la fuerte regresión marina acaecida hace aproximadamente 11 millones de años (Haq et al., 1988) para cruzar el estrecho de Bering como ya hizo anteriormente su predecesor Anchitherium. Con Hipparion llega una nueva gran oleada de inmigrantes, registrándose los primeros verdaderos jiráfidos, los grandes félidos machairodontinos, los úrsidos modernos (Agustí et al., 1997; Fraile et al., 1997) y las formas de rinocerontes típicas del final del Mioceno y el Plioceno (Cerdeño, 1992). Todas estas especies no desplazan a las especies preexistentes, sino que durante algún tiempo se añaden dando lugar al periodo de máxima riqueza del Neógeno. No obstante, algunas lineas muy características de tiempos anteriores desaparecen en este momento, como los paleomerícidos o los hemicyonidos, supervivientes del inicio del Mioceno. Como ya ocurriese en el aumento registrado durante la MN 4, se superponen dos circustancias en el fuerte aumento de la riqueza regional de la Península, por una parte, la apertura de vías migratorias y por otro una fuerte diferencia ambiental entre las cuencas, con la cuenca del Vallés-Penedés fuertemente segregada respecto al resto de las cuencas (Morales et al., 1999). Resulta notable la abundancia de taxa propios de ambientes húmedos y cálidos, tales como los tapires o los primates que han llevado a diversos autores a proponer un periodo de condiciones tropicales en España, en fuerte contraste con el deterioro climático registra- 
do a nivel global con el establecimiento del casquete polar antártico (Zachos et al., 2001) y el aumento de aridez inferido para la Europa meridional en este mismo periodo (Jernvall \& Fortelius, 2002). La Península parece haberse convertido en un refugio de las faunas más tropicales, con los máximos de riqueza de especies por yacimiento registrándose también en esta biozona.

La biozona MN 10 (9,5 a 9 Ma) mantiene un registro similar al observado en la biozona anterior aunque con un peor muestreo de la riqueza regional como muestran las cuervas de rarefacción. En esta biozona se aprecia una disminución de la riqueza peninsular que pudiera estar causada por este menor conocimiento aunque parece reflejar la paulatina desaparición de especies supervivientes del Mioceno medio, especialmente de aquellas más ligadas a hábitats boscosos y húmedos como los ciervos muntiacinos (Azanza et al., 1997) y diversas formas de suidos (Made, 1997). Desaparece asimismo la última especie de rinoceronte de talla pequeña (Cerdeño \& Nieto, 1995) y nuevas formas de hiénidos de talla grande, inmigrantes orientales, reemplazan a las especies previas de pequeña talla como Protictitherium (Agustí, 1999, ver también Fraile et al., 1997). La riqueza de especies en los yacimientos es aún de las más altas del Neógeno en lo que respecta a los ungulados, no así la total, aunque es posible que sea debido a falta de datos acerca de los carnívoros de esta época. La MN 10 marca el inicio de un declive de la riqueza de especies que va a continuarse, de forma irregular, a lo largo de todo el Mioceno superior y el Plioceno.

La MN 11 marca el inicio de unas nuevas faunas, mucho más adaptadas a ambientes áridos y mucho más empobrecidas. El registro de esta biozona es bastante pobre en número de yacimientos, que, sin embargo, están bien conocidos y repartidos en las cuencas más representativas. Ello parece indicar que el descenso en el número de especies presentes en la Península no está causado por la falta de registro, aunque no se puede obviar definitivamente esta posibilidad, sino que refleja un descenso real de la riqueza regional o $\gamma$-diversidad. Así, la riqueza de especies por yacimiento, tanto total como de ungulados, también disminuye fuertemente, con asociaciones dominadas por una única especie del équido Hipparion y por el bóvido Tragoportax (Morales et al., 1999). El aumento de la aridez y su efecto sobre la fauna se aprecia en la definitiva desaparición de las especies de ambientes húmedos, ya en declive en la biozona anterior, y por la entrada de una serie restringida de especies fuertemente ligadas a ambientes más secos incluso entre los cérvidos, con la entrada de formas de Cervinae (Azanza et al., 1997). Esta biozona ha sido señalada anteriormente como el momento de la instauración de un biotopo de tipo sabana en toda Europa (Morales \& Nieto, 1997). Una consecuencia de esta extensión general de los ecosistemas abiertos es, además de la pérdida de riqueza comentada, la homogeneidad en la fauna peninsular, con mínimas diferencias en las especies presentes en las distintas cuencas (Morales et al., 1999). El efecto de la extensión de la sabana se manifiesta en la siguiente biozona, la MN 12, cuando la riqueza aumenta con la incorporación de especies propias de este tipo de ambiente. Los bóvidos antilopinos e hipotraginos aportan el mayor número de especies a la fauna peninsular y, junto con los caballos, son los más abundantes en número de individuos por yacimiento (Nieto et al., 1997; Agustí, 1999). Este aumento del número de especies se refleja tanto en los yacimientos individuales como en el conjunto de la Península, a pesar de existir únicamente registro de dos cuencas y sólo una de ellas bien muestreada.

El final del Mioceno (MN 13; alrededor de 7 a 5,3 Ma) está marcado por la crisis de salinidad del Mesiniense, una fuerte regresión marina en el Mediterráneo que implicó la apertura de nuevas vías de comunicación con el continente Africano (Agustí \& Lleras, 1996; Agustí, 1999). Fruto de esta comunicación es la entrada de hipopótamos, macacos, félidos modernos (género Felis), camellos y algunos bóvidos asociados a ambientes húmedos (Morales y Nieto, 1997). Todos estos inmigrantes marcan un aumento del número de especies que, sin embargo, no se ve refrendado por una riqueza alta en los yacimientos. En este sentido, es posible que la diferencia de riqueza presente en la Península entre esta biozona y la previa MN 12 pueda estar causada por la presencia de registro en la cuenca levantina, ausente en la biozona anterior. Este aspecto es dificil de precisar, pero, sin embargo, sí permite reconocer una mayor diferencia en las condiciones ambientales presentes en las cuencas, con la cuenca levantina más húmeda, como se desprende de la mayor abundancia de cérvidos y bovinos forestales frente a la cuenca de Calatayud-Teruel, con una fauna más propia de hábitats abiertos y predominio de varias especies de caballos y de los antílopes dentro de los artiodáctilos.

El Plioceno (entre aproximadamente 5,3 y 1,9 Ma) está caracterizado por un importante descenso de la riqueza tanto en los yacimientos como en el conjunto de la Península. En sus inicios se establece la morfología actual del Mediterráneo con la transgresión pliocena (Rögl, 1999). Desde su ini- 
cio, en la MN 14, se produce la regresión de la mayor parte de las especies más ligadas a condiciones húmedas como los grandes herbívoros. Desaparecen asimismo una parte importante de los carnívoros preexistentes, sustituidos parcialmente por formas modernas de los géneros Hyaena, Lynx y Panthera (Fraile et al., 1997). En esta primera biozona del Plioceno, el número de especies en los yacimientos alcanza valores mínimos. La pobreza del registro en nuestra península durante el Plioceno puede tener un efecto importante sobre las estimaciones de la riqueza, como se puede apreciar en las curvas de rarefacción (Figura 4). En el caso de la MN 14, se añade el hecho de que sólo se dispone de registro de una cuenca interior, la de Calatayud-Teruel, de condiciones ambientales típicamente áridas a lo largo del tiempo. Sin embargo, aún dentro de la misma cuenca, el descenso en el número de especies es evidente e importante respecto a tiempos precedentes. En la siguiente biozona (MN 15; 4,2-3,4 Ma) continúa el descenso de la riqueza a pesar de contar con yacimientos en dos cuencas (una de ellas sin yacimientos bien muestreados) y con mayor riqueza de especies en cada yacimiento. El dominio de las faunas por el antílope Gazella borbonica indica el aumento paulatino de la aridez (Morales \& Nieto, 1997). Esta tendencia al aumento de la aridez se rompe en la última mitad del Plioceno (biozonas MN 16 y 17; 3,4-1,9 $\mathrm{Ma}$ ), caracterizada por un cambio en las condiciones ambientales, con descenso de las temperaturas y establecimiento del casquete polar ártico (Zachos et al., 2001). Se puede considerar este periodo como de transición hacia el clima propio del Pleistoceno. Este cambio se aprecia fundamentalmente por la entrada de nuevos rumiantes que se añaden a las empobrecidas faunas precedentes. Entre los inmigrantes destaca la entrada de cabras, ovibovinos, el género Cervus y, sobre todo, del mamut meridional (Morales \& Nieto, 1997). El resultado es una fauna algo más rica que las precedentes, con yacimientos ligeramente más ricos que al inicio del Plioceno, aunque sin aproximarse a los valores alcanzados en el Mioceno medio y superior.

\section{PATRONES GENERALES DE DIVERSIDAD}

Los procesos que producen cambios en los valores de diversidad dependen del enfoque, local o regional, que utilicemos (ver discusión en Ricklefs \& Schluter, 1993). En el caso de la diversidad local, los aumentos se pueden producir por dos vías fundamentales: i) desde arriba, mediante el efecto de procesos biogeográficos, que generan una mayor diversidad regional debido a la inmigración de fau- nas (a mayor disponibilidad de especies diferentes en el pool regional, mayor número de ellas se podrán asentar en un área determinada); ii) desde abajo, debido a factores ecológicos locales, como la productividad del ecosistema, el clima o procesos de estructuración de las comunidades. Del mismo modo, el efecto inverso (generar altos valores de diversidad regional a partir de efectos a escalas inferiores) puede ocurrir, a partir de comunidades locales tanto ricas como pobres. En este caso es la $\beta$-diversidad la responsable de facilitar una gran $\gamma$ diversidad; los procesos generadores de heterogeneidad ambiental, tanto espacial como temporal, juegan aquí un papel muy importante, permitiendo el asentamiento en la Península de diferentes faunas, y, por lo tanto, un mayor recambio de especies entre las diferentes cuencas.

En este sentido, las variaciones de la diversidad registradas en el Neógeno pueden resumirse en un aumento desde su inicio hasta el Mioceno superior, hace $10 \mathrm{Ma}$, con dos aumentos importantes en el inicio del Mioceno hace 18 millones de años y otra importante subida hace $11 \mathrm{Ma}$. Estos aumentos parecen estar relacionados con un incremento del pool regional en momentos de intensa migración posiblemente causadas por la apertura de rutas anteriormente cerradas, aunque sea parcialmente. Este mismo comentario puede aplicarse al aumento de la $\gamma$-diversidad registrado hace $6 \mathrm{Ma}$, durante la MN 13, asociable a la apertura de rutas migratorias debido a la crísis mesiniense y la consiguiente regresión del Mediterráneo. Desde el máximo alcanzado se inicia un descenso paulatino con valores mínimos hace $8 \mathrm{Ma}$ y alrededor de los 4 $\mathrm{Ma}$, en ambos casos coincidentes con el establecimiento de condiciones aparentemente más áridas. En ambos casos, tras el fuerte descenso causado por el cambio de condiciones ambientales se produce un cierto repunte en la riqueza cuando se establecen comunidades más propias de estas condiciones (por ejemplo en la MN 12). La $\alpha$-diversidad sigue un patrón similar pero con variaciones menos marcadas, apreciable fundamentalmente en los análisis con datos de ungulados. Existen, no obstante, algunas discrepancias en las tendencias como la registrada al final del Mioceno, hace 6 $\mathrm{Ma}$, cuando se alcanza un punto de elevada $\gamma$ diversidad con comunidades locales, sin embargo, empobrecidas respecto al periodo anterior. Esto implica un elevado recambio de especies que puede explicarse por una mayor heterogeneidad ambiental, mientras que la $\alpha$-diversidad se mantiene baja. Se han propuesto diferentes mecanismos capaces de producir patrones similares en comuni- 


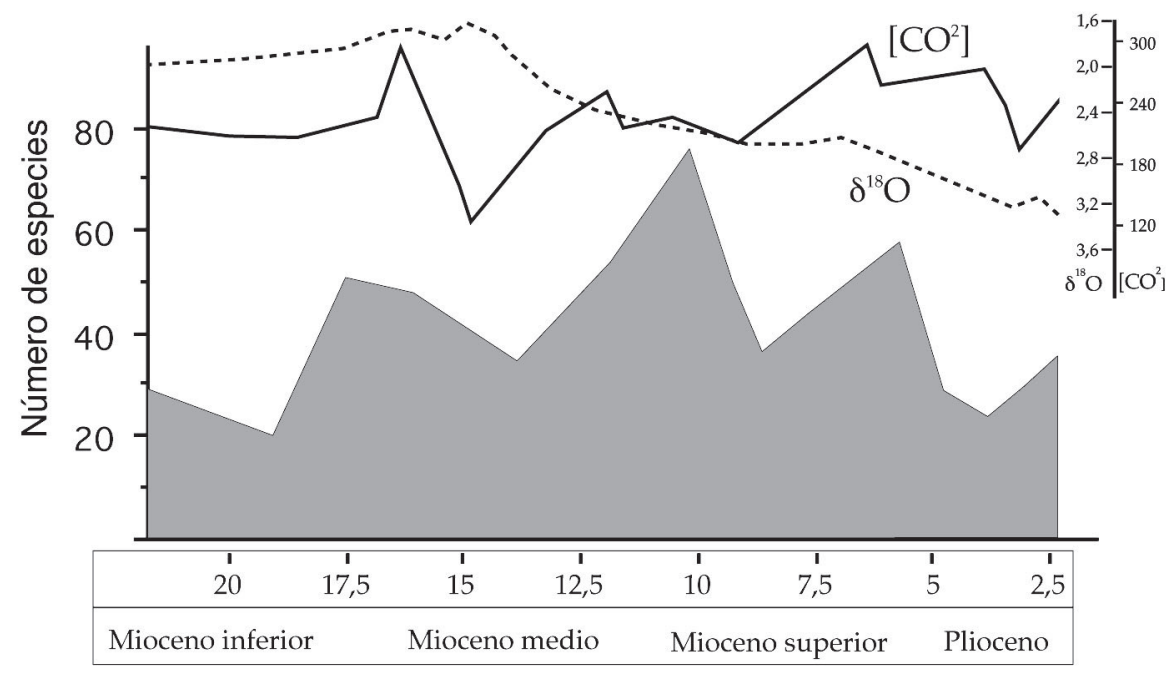

Fig. 7.- Variaciones en la riqueza de grandes mamíferos en las distintas biozonas del Neógeno de España comparadas con los cambios ambientales globales. Curva isotópica del oxígeno según Zachos et al. (2001). Curva de concentración del dióxido de carbono según Pearson \& Palmer (2000).

Fig. 7.- Changes in macromammal richness across the Neogene stages in Spain compared to global changes. Oxigen isotope curve modified from Zachos et al (2001). Carbon dioxide concentration curve based on Pearson \& Palmer (2000).

dades actuales, como una reducida productividad y/o un elevado estrés ambiental (cuyo efecto es difícil que se produzca sobre las comunidades muestreadas por un yacimiento durante largos periodos de tiempo). Sin embargo, recientes análisis sobre los determinantes de la variación a gran escala en la riqueza local de especies de mamíferos realizados con comunidades actuales muestran la gran importancia de efectos no ambientales (es decir, históricos y/o biogeográficos sensu lato) en la diversidad local (J. Hortal, J. Rodríguez, M. Nieto y J. M. Lobo, datos no publicados). Estos estudios muestran que la desaparición de unas pocas especies del pool regional de Sudamérica provoca una disminución general de la riqueza en las comunidades locales de la región Neotropical frente a las de la región Etiópica, que cuenta con un pool regional similar en número, así como una superficie y disposición geográfica similar. Esta disminución es debida a que los elementos que faltan en Sudamérica son grandes mamíferos, especies generalistas y de amplia distribución, presentes, por tanto, en casi todas las comunidades locales. Empobrecimientos similares en el pool regional producidos en especies de distribución restringida no tendrían un efecto marcado sobre el total de las comunidades locales, restringiéndose a los hábitats donde se habrían producido dichas extinciones. Por ello, para determinar las causas del tránsito entre $\alpha$ y $\gamma$ diversidades (es decir, la importancia de la $\beta$-diversidad) es necesario un análisis más exhaustivo de la composición de las comunidades originales y, consecuentemente, de las asociaciones encontradas en cada yacimiento y los procesos tanto tafonómicos como de muestreo que puedan sesgar dichas asociaciones.

La relación entre los cambios en las faunas de mamíferos con los cambios climáticos ha sido demostrada reiteradas veces. Los cambios ambientales globales para el Neógeno se pueden observar en la figura 7. Presentan un patrón caracterizado térmicamente por un periodo de altas temperaturas a comienzos del Mioceno con un máximo entre los 17 y $15 \mathrm{Ma}$ (el denominado optimo climático del Mioceno medio). A partir de este momento se produce un progresivo enfriamiento del planeta, con reestablecimiento del Casquete Antártico hace 10 $\mathrm{Ma}$, sólo interrumpido por un periodo de leve calentamiento a comienzos del Plioceno (5-3 Ma) antes del fuerte descenso de finales de este periodo cuando se forma definitivamente el casquete polar ártico (Zachos et al., 2001). Otro parámetro 
comúnmente estimado ha sido la concentración de $\mathrm{CO}_{2}$, de gran importancia para la productividad vegetal. Los recientes estudios realizados por Pearson \& Palmer (2000) muestran un importante descenso del $\mathrm{CO}_{2}$ al inicio del Mioceno y la presencia de bajas concentraciones durante todo el Mioceno inferior. Hace aproximadamente 16 Ma se produce un fuerte incremento seguido por otra no menos fuerte disminución de la concentración del $\mathrm{CO}_{2}$ hasta un mínimo en los 14,5 Ma. Desde este mínimo se produce un aumento hasta valores similares a los observados en el Mioceno inferior que se mantendran hasta los 9 Ma. En la parte final del Mioceno se registran altos valores que dan paso, en el final del Plioceno a un nuevo descenso en la concentración de $\mathrm{CO}_{2}$, hasta valores similares a los del inicio del Mioceno y el Mioceno superior. La relación de estos cambios con la riqueza de macromamíferos es irregular. En lo que respecta a la temperatura, se aprecia una cierta coincidencia del óptimo climático registrado entre 17 y 15 Ma con el primer fuerte aumento de la riqueza de la Península Ibérica, tanto a nivel de $\gamma$-diversidad como en $\alpha$-diversidad. Sin embargo, el subsiguiente descenso de la temperatura hasta los 10 millones de años no coincide con un patrón de cambio asimilable en la fauna, como tampoco los restantes cambios presentes. Sólo al final del Plioceno, la entrada de las faunas de clima frio que caracterizará al Pleistoceno refleja de manera fiel el fuerte descenso de la temperatura registrado globalmente (Zachos et al., 2001). En lo que respecta a los cambios en la concentración de $\mathrm{CO}_{2}$ sí parece haber una relación más directa con la riqueza de mamíferos (figura 7). El incremento registrado en la riqueza por yacimientos (local) y en el conjunto de la Península (regional) hace $17 \mathrm{Ma}$ coincide a grandes rasgos con el aumento de concentración de $\mathrm{CO}_{2}$ registrado por Pearson \& Palmer (2000), al igual que el descenso siguiente coincide con una menor riqueza regional (aunque no local) en la biozona MN 6 (hace $14 \mathrm{Ma}$ ). Algo similar se observa en el Mioceno superior, con aumentos y disminuciones coincidiendo de forma general. Así, el incremento de la riqueza regional y local registrado hace $11 \mathrm{Ma}$ coincide con un periodo de niveles altos de $\mathrm{CO}_{2}$ y el subsiguiente descenso coincide con un mínimo, aunque no marcado, en la concentración del $\mathrm{CO}_{2}$. Finalmente la riqueza regional registrada al final del Mioceno coincide con un máximo de $\mathrm{CO}_{2}$ atmosférico seguido por un importante descenso también apreciable en la fauna.

Un aspecto especialmente relevante de los patrones obtenidos es la presencia de un periodo de elevada $\alpha$-diversidad entre los 12 y los 6 Ma para los ungulados y más restringido, entre 10 y $8 \mathrm{Ma}$, para el conjunto de los macromamíferos (figura 2). En ambos casos, la riqueza local de especies observada en los yacimientos supera los máximos observados en comunidades actuales de latitudes no tropicales (datos en Rodríguez y Nieto, en preparación). Estas comparaciones son, por supuesto, preliminares por cuanto las comunidades actuales pueden estar (y de hecho en muchos casos lo están) empobrecidas por efectos antrópicos y las faunas de yacimientos individuales como estimadores de la $\alpha$-diversidad pueden estar fuertemente sesgadas tafonómicamente. Sin embargo, todo ello no debe oscurecer el hecho de que durante el Mioceno medio y el inicio del Mioceno superior se registran una serie de faunas de alta riqueza local. Tal y como hemos comentado anteriormente, dos aspectos parecen determinar la diversidad: procesos biogeográficos y factores ambientales. De entre estos últimos, la productividad ha sido señalada como uno de los principales factores en las comunidades actuales (Rosenzweig, 1995) y en las fósiles (Janis et al., 2000 y 2002). Los resultados obtenidos son, hasta cierto punto, corcondantes con los presentados por Janis et al. $(2000,2002)$ para los ungulados de la región de Nebraska (U.S.A.) aunque el máximo de diversidad se alcanza antes en Norteamérica (Mioceno inferior y medio; 15 y 12 Ma). La interpretación de las faunas llevó a Janis et al. $(2000,2002)$ a proponer un periodo global de alta productividad, ligado a un aumento en la concentración de $\mathrm{CO}_{2}$, durante el Mioceno inferior y medio. Esta posibilidad se ve apoyada en lo referente a las faunas españolas por la correlación, previamente comentada, entre los momentos de máxima diversidad y concentraciones altas de $\mathrm{CO}_{2}$. Sin embargo, el diacronismo de los máximos presentes en España y Norteamérica parece contradecir el carácter global del proceso, tal y como lo proponen Janis y colaboradores (op. cit.), apuntado hacia factores no globales, como los que produjeron la expansión de los ecosistemas herbáceos en las distintas partes del mundo (Janis, 1984).

\section{Conclusiones}

Los análisis realizados el presente trabajo muestran cambios importantes en la riqueza local $(\alpha-$ diversidad) y regional ( $\gamma$-diversidad) a lo largo del registro ibérico del Neógeno. La interpretación de estos cambios ha de llevarse a cabo con cautela dado que, como se ha demostrado, una parte importante de la variabilidad observada en la riqueza regional está causada por diferencias en el mues- 
treo, tanto en la cantidad de yacimientos estudiados (y su calidad) -como se demuestra en las curvas de rarefacción- como por diferencias en el área con registro conocido en las distintas biozonas. Los análisis que se han realizado, hasta cierto punto preliminares, indican la necesidad de profundizar en el modo de cuantificar de la diversidad del pasado, analizando en detalle los efectos de las diferencias en el muestreo, tanto a nivel de yacimientos como de áreas incluidas.

Al analizar el registro del Neógeno en la Península, teniendo en cuenta las limitaciones señaladas anteriormente, se aprecia la existencia de un patrón general, hasta cierto punto paralelo, en los cambios de la riqueza local y peninsular, con valores altos durante el final del Mioceno medio y el inicio del Mioceno superior (entre 12 a $6 \mathrm{Ma}$. con cénit entre 10 y $8 \mathrm{Ma}$ ), y valores más bajos durante el Mioceno inferior y el final del Mioceno y el Plioceno. A pesar de esta coincidencia general, los cambios en ambas propiedades presentan diferencias muy marcadas. La riqueza general de la Península ( $\gamma$-diversidad) presenta tres momentos de fuerte aumento localizados en las MN 4, 10 y 13 todos ellos coincidentes con eventos geográficos que posibilitan la entrada de nuevos elementos faunísticos que se unen a los previamente registrados. Estos cambios, sin embargo, no tienen su correspondencia temporal en la $\alpha$-diversidad (riqueza local), cuyos valores no parecen depender directamente de dichos cambios. Los cambios de la riqueza local de especies son más difícilmente interpretables, pudiendo estar relacionados con cambios en la productividad primaria (ligados a cambios en la concentración de $\mathrm{CO}_{2}$ atmosférico) aunque también pueden depender de la riqueza peninsular que constituye su pool regional.

Asimismo, el presente estudio muestra una importante disparidad entre las faunas de las diferentes cuencas registradas en la Península, fundamentalmente entre las cuencas costeras (VallésPenedés y Levantinas) frente a las interiores (Tajo y Calatayud-Teruel). Esta heterogeneidad espacial, previamente apuntada por Morales et al. (1999) y Agustí (1999), está causada por diferencias ambientales más o menos acusadas en las distintas biozonas.

\section{AGRADECIMIENTOS}

En primer lugar agradecer a J. Lobo su amable invitación a colaborar en este homenaje. Gracias también a Yolanda Fernández Jalvo por sus comentarios y discusión crítica del presente trabajo. Este estudio ha sido posible gracias a los proyec- tos de investigación PB98-0513, MCYT BX X 2000-1258CO3-01, BTE 2002-00410, REN 2001-1136/GLO y las becas predoctorales del MNCN/CSIC/Comunidad de Madrid (J.H.) y del Colegio de España (C.M.).

\section{Referencias}

Aguirre, E. \& RÁBAno, I., 1999. La huella del pasado: fósiles de Castilla-La Mancha. Patrimonio histórico de Castilla-La Mancha, 16. Junta de Comunidades de Castilla-La Mancha, Toledo. 381 pp.

Agustí, J., 1999. A critical re-evaluation of the Miocene mammal units in Western Europe: dispersal events and problems of correlation. In: J. Agustí, L. Rook \& P. Andrews (eds.). The evolution of Neogene Terrestrial Ecosystems in Europe. Cambridge University Press. Cambridge: 84-112.

Agustí, J., Cabrera, L., Garcés, M. \& PArés, J. M., 1997. The Vallesian mammal succession in the Vallés-Penedés basin (northeast Spain): paleomagnetic calibration and correlation with global events. Paleogeography, Paleoclimatology, Paleoecology, 133(3-4): 149-180.

Agustí, J. \& LlenAs, M., 1996. The late Turolian muroid rodent succession in eastern Spain. Acta Zoologica Cracoviensia, 39(1): 47-56.

AlBerdi, M. T., 1989. A review of old World hipparionine horses. In: D. R. Prothero \& R. M. Schoch (eds.). The evolution of Perissodactyls. Oxford University Press. Oxford: 234-261.

Alberdi, M. T. \& Rodríguez, J., 1999. Restos de Anchitherium de Lisboa, Portugal. Cienciâs da Terra 13: 93-114.

AlcalÁ, L., 1994. Macromamíferos Neógenos de la Fosa de Teruel-Alfambra. Instituto de Estudios Turolenses-Museo Nacional de Ciencias Naturales. Teruel. 554 pp.

Alcalá, L., Cerdeño, E., Montoya, P., Morales, J., PÉrez, B. \& SoriA, D., 1990. Composición taxonómica y anatómica de los restos de macrovertebrados del Mioceno inferior continental de Loranca del Campo (Cuenca). Comunicaciones de la Reunión de Tafonomía y Fosilización, Madrid: 7-12.

Alroy, J., 1996. Constant extinction, constrained diversification, and uncoordinated stasis in North American mammals. Palaeogeography, Palaeoclimatology, Palaeoecology, 127: 285-311.

Andrews, P., Lord, J. M. \& Nesbit-Evans, E. M., 1979. Patterns of diversity in fossil and modern mammalian faunas. Biological Journal of the Linnean Society, 11: 177-205.

Azanza, B., Nieto, M., Soria, D. \& Morales, J., 1997. El registro fósil neógeno de los Cervoidea (Artiodactyla, Mammalia) de España. In: J. P. Calvo \& J. Morales (eds.). Avances en el Conocimiento del 
Terciario Ibérico. Universidad Complutense-CSIC. Madrid: 41-44.

Cerdeño, E., 1992. Spanish Neogene rhinoceroses. Palaeontology, 35: 297-308.

Cerdeño, E. \& Nieto, M., 1995. Changes in Western European Rhinocerotidae related to climatic variations. Palaeogeography, Palaeoclimatology, Palaeoecology, 114: 325-338.

Colwell, R. K., 2000. EstimateS 6.01b. Programa y manual disponibles en: http://viceroy.eeb.uconn.edu/Estimates6/.

Colwell, R. K. \& Coddington, J. A., 1994. Estimating terrestrial biodiversity through extrapolation. Philosophical Transactions of the Royal Society of London B, 345: 101-118.

Costa Tenorio, M., Morla Juaristi, C. \& SÁinz Ollero, H. (eds.). 1998. Los Bosques Ibéricos. Una interpretación geobotánica. Planeta. Barcelona. 572 pp.

Dam, J. van, Alcalá, L., Alonso Zarza, A., Calvo, J. P., GARcÉs, M. \& KRIJgSman, W,. 2001. the Upper Miocene Mammal record from the Teruel-Alfambra region (Spain). The MN system and continental stage/age concepts discussed. Journal of vertebrate Paleontology, 21(2): 367-385.

Fraile, S., Pérez, B., Miguel, I. \& Morales, J., 1997. Revisión de los carnívoros presentes en los yacimientos del Neógeno español. In: J. P. Calvo y J. Morales (eds.). Avances en el Conocimiento del Terciario Ibérico. Universidad Complutense-CSIC. Madrid: 137-140.

Garcés, M., Cabrera, L., Agustí, J. \& Parés, J. M., 1997. Old World first appearance datum of "Hipparion" horses: late Miocene large mammal dispersal and global events. Geology, 25(1): 19-22.

Gotelli, N. J. \& Colwell, R. K., 2001. Quantifying biodiversity: procedures and pitfalls in the measurement and comparison of species richness. Ecology Letters, 4: 379-391.

HaQ, B. U., Hardenbol, J. \& Vail, P. R., 1988. Mesozoic and Cenozoic chronostratigraphy and cycles of sea-level changes. In: C. K. Wilgus et al. (eds.). Sea-level Changes - an Integrated Approach. SEMP Special Publication 42: 71-108.

HorTAL, J. \& LoBo, J. M., 2002. Una metodología para predecir la distribución espacial de la diversidad biológica. Ecología (n.s.), 16: 151-178.

JANIS, C. M., 1984. The use of fossil ungulate communities as indicators of climate and environment. In: P. Brenchley (ed.). Fossils and Climate. Wiley. New York: 85-103.

Janis, C. M., Damuth, J. \& Theodor, J. M., 2000. Miocene ungulates and terrestrial primary productivity: Where have all the browsers gone? Proceedings of the National Academy of Sciences of the United States of America, 97(14): 7899-7904.
JANIS, C. M., DAMUth, J. \& ThEODOR, J. M., 2002. The origins and evolution of the North American grassland biome: the story from the hoofed mammals. Palaeogeography, Palaeoclimatology, Palaeoecology, 177: 183-198.

Jernvall, J. \& Fortelius, M., 2002. Common mammals drive the evolutionary increase of hypsodonty in the Neogene. Nature, 417: 538-540.

Koleff, P. \& Gaston, K. J., 2002. The relationships between local and regional species richness and spatial turnover. Global Ecology and Biogeography, 11: 363-375.

MAde, J. VAN DER, 1997. Los suoidea de la Península Ibérica. In: J. P. Calvo y J. Morales (eds.). Avances en el Conocimiento del Terciario Ibérico. Universidad Complutense-CSIC. Madrid: 109-112.

Mazo, A. V., 1977. Revisión de los mastodontes de España. Tesis Doctoral. Universidad Complutense de Madrid. 440 pp.

MeIN, P. 1975. Proposition de biozonation du Néogène Méditerranéen a partir des mammifères. Trabajos de Neógeno y Cuaternario, 4: 112.

Meulen, A. VAN DER \& DAAMS, R., 1992. Evolution of early-middle Miocene rodent faunas in relation to long term palaeoenvironmental changes. Palaeogeography, Palaeoclimatology, Palaeoecology, 93: 227-253.

Morales, J., Alcalá, L., Hoyos, M., Montoya, P., Nieto, M., Pérez, B. \& SoriA, D., 1993. El yacimientos del Aragoniense medio de La Retama (Depresión Intermedia, provincia de Cuenca, España): significado de las faunas con Hispanotherium. Scripta Geologica, 103: 23-39.

Morales, J. \& Nieto, M., 1997. el registro terciario y cuaternario de los mamíferos de España. In: J. P. Calvo y J. Morales (eds.). Registros fósiles e Historia de la Tierra. Editorial Complutense. Madrid: 297-322.

Morales, J., Nieto, M., Amezua, L., Fraile, S., Gómez, E., Herráez, E., Peláez-Campomanes, P., Salesa, M. J., SÁnchez, I. M. \& Soria, D., 2000. El patrimonio paleontológico de la Comunidad de Madrid. Arqueología, Paleontología y Etnografía 7. Editorial de la Comunidad Autónoma de Madrid, Madrid. 389 pp.

Morales, J., Nieto, M., Khöler, M. \& MoyÁ-SolÁ, S., 1999. Large mammals from the Vallesian of Spain. In: J. P. Calvo y J. Morales (eds.). The evolution of Neogene Terrestrial Ecosystems in Europe. Cambridge University Press. Cambridge: 113-126.

Morales, J., Pickford, M. \& Soria, D., 1993. Pachyostosis in lower Miocene giraffoid from Spain Lorancameryx pachyostoticus nov. gen. nov. sp. and its bearing on the evolution of bony appendages on artiodactyls. Geobios, 26: 207-230. 
MoyÁ-SolÁ, S. \& Agustí, J., 1990. Bioevents and mammal successions in the Spanish Miocene. In: E. H. Lindsay, V. Fahlbusch \& P. Mein (eds.). European Neogene Mammal Chronology. Plenum Press. New York: 357-373.

Nieto, M., Azanza, B., Soria, D. \& Morales, J., 1997. El registro fósil neógeno de los Bovoidea (Artiodactyla, Mammalia) de España. In: J. P. Calvo y J. Morales (eds.). Avances en el Conocimiento del Terciario Ibérico. Universidad Complutense-CSIC. Madrid: 137-140.

Pearson, P. N. \& Palmer, M. R., 2000. Atmospheric carbon dioxide concentrations over the past 60 million years. Nature, 406: 695-699.

PiCKFoRD, M. \& Morales, J., 1994. Biostratigraphy and palaeobiogeography of East Africa and the iberian peninsula. Palaeogeography, Palaeoclimatology, Palaeoecology, 112: 297-322.

Ricklefs, R. E. \& Schluter, D. (eds.), 1993. Species Diversity in Ecological Communities. Historical and Geographical Perspectives. The University of Chicago Press. London. 416 pp.

Rodríguez, J. \& Nieto, M., En preparación. Paleoclimatic inferencies from fossil mammal assemblages: Theoretical constraits and practical limitations.

RöGL, F., 1999. Mediterranean and Paratethys palaeogeography during the Oligocene and Miocene. In: J. Agustí, L. Rook \& P. Andrews (eds.). The evolution of Neogene Terrestrial Ecosystems in Europe. Cambridge University Press. Cambridge: 8-22.

RosenZweig, M. L., 1995. Species diversity in Space and Time. Cambridge University Press. Cambridge, UK. $458 \mathrm{pp}$.

SÁnchez, I. M., Salesa, M. J. \& Morales, J., 1998. Revisión sistemática del género Anchitherium Meyer 1834 (Equidae; Perissodactyla) en España. Estudios Geológicos, 54(1-2): 39-63.

SANZ DE SIRIA, A., 1985. La flora miocénica de los alrededores de Sant Sadurni d'Anoia (Barcelona). Paleontologia i Evolució, 18: 161-172.

SANZ DE SIRIA, A., 1988. Los vegetales miocéncos de Rubí (Barcelona). Paleontologia i Evolució, 22: 71-76.
SANZ DE SiRIA, A., 1994. La evolución de la paleofloras en las cuencas cenozoicas catalanas. Acta Geológica Hispánica, 29(2-4): 169-189.

SEN, S., 1990. Hipparion datum and its chronologic evidence in the Mediterranean area. In: E. H. Lindsay, V. Fahlbusch \& P. Mein (eds.). European Neogene Mammal Chronology. Plenum Press. New York: 495-505.

Soberón, J. \& Llorente, J., 1993. The use of species accumulation functions for the prediction of species richness. Conservation Biology, 7(3): 480-488.

STATSOFT, 2001. STATISTICA (data analysis software system) version 6.0. StatSoft, Inc., Tulsa, OK.

Steininger, F. F., Berggren, W. A., Kent, D. V., Bernor, R. H., Sen, S. \& Agustí, J., 1996. CircumMediterranean Neogene (Miocene-Pliocene) marinecontinental chronological correlations of European mammal units. In: R. L. Bernor, V. Fahlbusch \& H.W. Mittmann (eds.). The evolution of Western Eurasian Neogene Mammal Faunas. Columbia University Press. New York: 7-46.

THENIUs, E., 1972. Grundzüge der Verbreitungsgeschichte der Säugertiere. G. Fisher Verlag. Jena. 345 pp.

VRBA, E. S., 1980. The significance of bovid remains as indicators of environmental and predation patterns. In: A. K. Behrensmeyer \& A. Hill (eds.). Fossils in the making: Vertebrate Taphonomy and Paleoecology. Chicago University Press. Chicago: 247-271.

Whitakker, R. H., 1960. Vegetation of the Siskiyou Mountains, Oregon and California. Ecological Monographs, 30: 279-338.

Whitakker, R. J., Willis, K. J. \& Field, R. 2001. Scale and species richness: towards a general, hierarchical theory of species richness. Journal of Biogeography, 28: 453-470.

Wing, S. L., Alroy, J. \& Hickey, L. J., 1995. Plant and mammal diversity in the Paleocene to early Eocene of the Bighorn Basin. Palaeogeography, Palaeoclimatology, Palaeoecology, 115: 117-155.

Zachos, J., Pagani, M., Sloan, L., Thomas, E. \& BiLluPs, K., 2001. Trends, Rhythms, and Aberrations in Global Climate 65 Ma to Present. Science, 292: 686-693. 